\title{
Generating neutron-star magnetic fields: three dynamo phases
}

\author{
S. K. Lander ${ }^{\star}$ \\ School of Physics, University of East Anglia, Norwich, NR4 7TJ, U.K.
}

25 July 2021

\begin{abstract}
Young neutron stars (NSs) have magnetic fields in the range $10^{12}-10^{15} \mathrm{G}$, believed to be generated by dynamo action at birth. We argue that such a dynamo is actually too inefficient to explain the strongest of these fields. Dynamo action in the mature star is also unlikely. Instead we propose a promising new precession-driven dynamo and examine its basic properties, as well as arguing for a revised mean-field approach to NS dynamos. The precession-driven dynamo could also play a role in field generation in main-sequence stars.
\end{abstract}

Key words: dynamo - stars: evolution - stars: magnetic fields - stars: neutron - stars: rotation

\section{INTRODUCTION}

The strongest long-lived magnetic fields $B$ in the Universe are hosted by neutron stars (NSs), with inferred external dipole fields $B_{\text {dip }}$ up to $10^{15} \mathrm{G}$ in strength, but usually considerably weaker. For older NSs some of this variability can be attributed to accretion or field evolution, but even among young NSs the variation is huge: for example, the Crab pulsar and the magnetar SGR 1806-20 are both around $1000 \mathrm{yr}$ old, but the former has $B_{\text {dip }}=3.8 \times 10^{12} \mathrm{G}$ and the latter $B_{\text {dip }} \approx(0.8-2) \times 10^{15} \mathrm{G}$. The physics that leads to some NSs - but not others - having $B_{\text {dip }} \sim 10^{15} \mathrm{G}$ is still poorly understood, despite being of interest both on theoretical grounds, and for observed astrophysical phenomena. For example, early generation of a strong $B$ is essential for leading models of superluminous supernovae, $\gamma$-ray bursts and afterglows (Zhang \& Mészáros 2001; Thompson et al, 2004), and might result in the star emitting detectable grayitational radiation (Stella et al. 2005).

The simplest explanation for NS magnetism is that it is a 'fossil', inherited from the degenerate iron core of its progenitor star. Magnetic flux scales with the square of the stellar radius $R_{*}$; assuming this is conserved in the compression of a typical progenitor $\left(R_{*} \sim 10^{6} \mathrm{~km}\right)$ to NS proportions $\left(R_{*} \sim 10 \mathrm{~km}\right)$ yields a factor- $10^{10}$ amplification of $B$. One can thus account for the highest $B$ in NSs, since there do indeed exist main-sequence stars with $B$ up to $\sim 10^{4}$ G (Landstreet 1992; Donati \& Landstreet 2009) - although the actual amplification factor will be less than $10^{10}$, since only the flux of the progenitor's iron core is inherited by the NS. Immediately before collapse this core has a radius $\sim 3000 \mathrm{~km}$ (Sukhbold et al. 2016), so within the fossil field scenario its $B$ would in some cases be as high as $(10 / 3000)^{2} \times 10^{15} \mathrm{G}=10^{10} \mathrm{G}$. NS magnetic fields are unlikely to be fossils, however, for at least two reasons: there appear to be too few suitable progenitors to explain the likely magnetar birth rate (Makarenko et al. 2021); and the violent dynamics in which a NS is born will not leave $B$ unaffected.

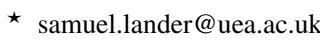

If $B$ is not a fossil, then, it must be amplified after birth to the kind of strengths we see. Large-scale efficient amplification of $B$ requires a dynamo: a mechanism for converting kinetic energy of (generally) small-scale fluid motions to magnetic energy (Moffatt 1978; Rincon 2019). NSs have extremely low electrical resistivity $\eta$, and so field lines are 'frozen' into the fluid. A dynamo exploits this: any field lines threading a fluid element are stretched as the fluid moves, thus increasing the magnetic energy. At the same time, this amplified and distorted field needs $\eta \neq 0$ in order to reconnect in its new geometry. We will show that reconnection is particularly difficult in the unique physical conditions of a NS.

Here we assess the possibility of dynamo action in a NS during three different phases of its life, with a view to understand how and when its intense $B$ is generated. We argue for a revision of dynamo theory for this problem, show how the usual scenario for magneticfield generation in NSs may not work, and present a promising new alternative.

\section{PHASE 1: THE HOT CONVECTIVE STAR}

In its very early life a NS experiences differential rotation (e.g. Janka \& Moenchmeyer (1989)), a consequence of the approximate conservation of angular momentum of cylindrical shells of matter during core collapse. In addition, its outer half will be convectively unstable (Epstein 1979). These are just the ingredients needed for dynamo action, with the prospect of amplifying the star's largescale $B$ up to $\sim 10^{15} \mathrm{G}$ by drawing on kinetic energy from turbulent motions (Thompson \& Duncan 1993).

A stellar dynamo involves dynamics over a wide range of lengthscales, from $R_{*}$ down to a typically microscopic scale related to e.g. damping/reconnection, making it intractable to study without some kind of approximation. One either attempts a highresolution direct numerical simulation, hoping that the unresolvable fine lengthscales are not essential for the dynamo, or uses lower resolution with a method to explicitly account for the effect of the subgrid physics. Studies of magnetic-field generation often employ 
mean-field theory (Krause \& Raedler 1980), an averaging procedure in which $\boldsymbol{B}$ and the velocity $\boldsymbol{v}$ are split into large-scale meanaveraged quantities, and small-scale fluctuating/stochastic terms. Only the former (here denoted with overbars) directly enter the fieldevolution equations, with the influence of the latter felt through a dynamo closure relation - essentially an assumption about the form the small-scale $\boldsymbol{v} \times \boldsymbol{B}$ term takes upon mean-averaging. In particular, turbulent convection is accounted for through an additional ' $\alpha$ effect' term in the mean- $B$ evolution:

$\frac{\partial \overline{\boldsymbol{B}}}{\partial t}=\nabla \times[\overline{\boldsymbol{v}} \times \overline{\boldsymbol{B}}+\alpha \overline{\boldsymbol{B}}-\eta \nabla \times \overline{\boldsymbol{B}}]$

(a similar result may be derived in general relativity; Bucciantini \& Del Zanna (2013)). B can be amplified through the joint action of turbulent convection with differential rotation - an ' $\alpha-\Omega$ dynamo' - or by convection alone through an ' $\alpha 2$ dynamo'. Evolving the mean-field equations, Bonanno et al. (2003) find that the dominant dynamo effect for a proto-NS seems to vary with rotation rate. Recent direct numerical simulations give additional information: that although the highest $B$ are generated in rapidlyrotating models (Raynaud et al. 2020), some dynamo activity is still present at slower rotation (Masada et al. 2020).

Two dimensionless numbers are key to understanding NS dynamos: the magnetic Reynolds and magnetic Prandtl numbers, $\mathrm{Rm}$ and $\operatorname{Pr}_{\mathrm{m}}$ respectively. $\mathrm{Rm} \equiv v_{\text {char }} l_{\text {char }} / \eta$ gives the ratio of advection to diffusion of $B$ by the flow (where $v_{\text {char }}$ and $l_{\text {char }}$ are the characteristic velocity and lengthscale of the flow), and $\operatorname{Pr}_{\mathrm{m}} \equiv v_{\mathrm{S}} / \eta$ shows the relative importance of kinematic shear viscosity $v_{\mathrm{S}}$ to resistivity in dissipating energy of the magnetised fluid. In a young $\mathrm{NS}^{1} \eta$ is primarily due to electron-proton scattering, with a typical value of $10^{-6}-10^{-4} \mathrm{~cm}^{2} \mathrm{~s}^{-1}$ (Baym et al. 1969; Raynaud et al. 2020). The main contribution to $v_{\mathrm{s}}$ changes depending on whether the stellar matter has cooled enough to be neutrino-transparent, a transition that occurs within a minute from birth. If so, neutron-neutron scattering dominates and $\approx 1 \mathrm{~cm}^{2} \mathrm{~s}^{-1}$ (Cutler \& Lindblom 1987); if not, neutrino-nucleon scattering dominates, leading to a much higher $v_{\mathrm{s}} \approx 10^{8} \mathrm{~cm}^{2} \mathrm{~s}^{-1}$ (Keil et al. 1996). The latter, neutrino-opaque, regime is relevant for a proto-NS, and so $\operatorname{Pr}_{\mathrm{m}} \sim 10^{13}$. To find Rm we follow Thompson \& Duncan (1993) and take $v_{\text {char }}=10^{8} \mathrm{~cm} \mathrm{~s}^{-1}, l_{\text {char }}=10^{5} \mathrm{~cm}$, yielding Rm $\sim 10^{17}$.

The best-understood dynamos are 'slow', with growth rates that tend to zero as $\mathrm{Rm} \rightarrow \infty$. 'Fast' dynamos, by constrast, still generate $B$ in this limit, even if they cannot be truly non-diffusive (Moffatt \& Proctor 1985); the archetypical example is the stretchtwist-fold dynamo (Vainshtein \& Zel'doyich 1972). Rigorous analysis is difficult, but any dynamo in a NS must - given their enormous $\mathrm{Rm}$ - be fast, so we will assume that results for both fast and high$\mathrm{Rm}$ dynamos (in principle distinct notions) are relevant here.

Any dynamo has to create magnetic flux more quickly than it is dissipated, suggesting that a large Rm is helpful - but the huge values associated with proto NSs in particular are, in fact, problematic. At least some such dynamos involve chaotic fluid motions that result in fractally-distributed $B$ with a strongly fluctuating direction (Finn \& Ott 1988); reconnection could then cause local cancellations of parallel and antiparallel field vectors, leaving a weak large-scale $B$. Indeed, Vainshtein \& Cattaneo (1992) found that high-Rm dynamos saturate at values of flux too low to explain typical astrophysical $B$. A related concern is how a large-scale $B$

1 Throughout this paper, unless stated otherwise, we report typical numerical values for a proto-NS core, with $\rho \approx 10^{14} \mathrm{~g} \mathrm{~cm}^{-3}, T \approx 10^{10}-10^{11} \mathrm{~K}$. can be rearranged, given that the low $\eta$ suggests a microscopic reconnection scale. This was allayed by Lazarian \& Vishniac (1999), who showed that high-Rm MHD turbulence with a weak stochastic component does allow for fast reconnection of the large-scale $B$ - and Parker (1992) argues that fast reconnection in turn supports a fast dynamo. Furthermore, an inverse cascade effect can convert small-scale helicity into large-scale $B$ (Frisch et al. 1975; Brandenburg 2001). Together, these studies give confidence in the ability of high-Rm dynamos to amplify large-scale $B$, and also suggest that numerical simulations of astrophysical dynamos - which necessarily employ unphysically small $\mathrm{Rm}$ - are nonetheless faithful to the astrophysical phenomena they intend to represent.

Key to these results, however, is that $\operatorname{Pr}_{m}$ is small, as is the case for non-degenerate stars but emphatically not for NSs. At large $\mathrm{Pr}_{\mathrm{m}}$ turbulence will tend to be viscously smoothed out on lengthscales longer than those on which reconnection takes place. This causes a reduction in reconnection speed by a factor (Jafari et al. 2018)

$$
\frac{\operatorname{Pr}_{\mathrm{m}}^{-1 / 2}}{1+\ln \left(\operatorname{Pr}_{\mathrm{m}}\right)}
$$

compared with the $\operatorname{Pr}_{\mathrm{m}}=1$ case; for a proto-NS the reduction factor is $10^{8}$, and the effect on dynamo action may be similarly deleterious. In addition, at large $\operatorname{Pr}_{\mathrm{m}}$ the inverse cascade effect is replaced by a 'reversed dynamo', in which conversion of magnetic to kinetic energy occurs at short lengthscales (Brandenburg \& Rempel 2019), potentially thwarting efficient large-scale field amplification.

Pessimistically, one could therefore envisage that whilst a real proto-NS dynamo amplifies a small-scale multidirectional $B$, this is then substantially annulled as it slowly reconnects, never managing to amplify the large-scale $B$. Furthermore, the work of Jafari et al. (2018) and Brandenburg \& Rempel (2019) suggests that typical proto-NS dynamo simulations - in which $\mathrm{Rm}, \mathrm{Pr}_{\mathrm{m}}$ are factors of $10^{16}, 10^{11}$ (respectively) too small - may not be representative of the real system ${ }^{2}$.

These issues would vex not only the convective dynamo, but also any other field-amplification mechanism during this phase: e.g. one driven by the magneto-rotational instability (Obergaulinger et al. 2009; Sawai et al. 2013; Mösta et al. 2015; Reboul-Salze et al. 2021) or the Tayler-Spruit dynamo (Spruit 2002).

How can we understand the details of a proto-NS dynamo, if realistic $\mathrm{Rm}$ and $\operatorname{Pr}_{\mathrm{m}}$ values are unattainable in a numerical approach? One possibility could be evolutions employing a revised mean-field dynamo that reflects the unique small-scale conditions of a high- $\mathrm{Rm}$, high- $\mathrm{Pr}_{\mathrm{m}}$ dynamo through a suitable closure relation. It is known that such conditions tend to produce a field concentrated into flux ropes (Galloway et al. 1978), which is analogous to a similar problem in the context of a mature NS core, where type-II superconductivity quantises the local field into thin fluxtubes. The global magnetic-field evolution of this latter problem has been studied in some detail (Mendell 1998; Graber et al. 2015), and provides a promising starting point for revising the NS dynamo equations.

\footnotetext{
2 Some such simulations (e.g. Mösta et al. (2015)) evolve the ideal MHD ( $\eta=0$ ) equations, relying on the unphysical artefact of numerical resistivity to provide reconnection. These 'ideal' simulations therefore have dissipation
} on the grid spacing $\left(10^{3}+\mathrm{cm}\right)$ and so an effective $\mathrm{Rm} \lll 10^{17}$. 


\section{PHASE 2: THE WARM PRECESSING STAR}

Precession was originally proposed as a possible mechanism for driving the geodynamo (Bullard 1949; Malkus 1968), and both numerical models (Tilgner 2005, 2007; Wu \& Roberts 2009) and laboratory experiments (Giesecke et al. 2018) have established its viability for amplifying $B$; in all cases a solid boundary precesses and drives internal fluid motion. Precession consists of a vector sum of rotations about two axes:

$\mathbf{\Omega}=\mathbf{\Omega}_{0}+\mathbf{\Omega}_{\mathrm{p}}$

where $\boldsymbol{\Omega}_{0}, \boldsymbol{\Omega}_{\mathrm{p}}$ are the primary and secondary rotations. In literature on fluid dynamics, the ratio of these two is often called the Poincaré number Po $=\Omega_{\mathrm{p}} / \Omega_{0}$, with a typical value being Po $=0.1$.

A NS can undergo free precession (i.e. no external driving force) due to the presence of a distortion misaligned from $\boldsymbol{\Omega}_{0}$ by some angle $\chi$. Often this is assumed to be an elastic asymmetry in the star's solid crust (Jones \& Andersson 2001), but by the time the star has cooled enough for this to form, dynamo action may well be totally suppressed, as discussed later.

Here we describe a new precession-driven dynamo that can operate in an entirely fluid body, applying the idea to a young NS. It uses the key result that the star's $B$ always induces some distortion (or 'rigidity') $\epsilon_{B} \propto B^{2}$ that is typically misaligned from $\boldsymbol{\Omega}_{0}$ by some angle $\chi$ and thus drives precession (Spitzer 1958).

A dominantly poloidal (toroidal) field induces an oblate (prolate) distortion. The two cases have different minimum-energy states: $\chi=0^{\circ}\left(90^{\circ}\right)$ for an oblate (prolate) body. Now, once the proto-NS phase has finished it is likely that differential rotation will have wound up the birth $B$ to leave a strong toroidal component $B_{\text {tor }}$ roughly symmetric about $\boldsymbol{\Omega}_{0}$ (i.e. $\chi \approx 0^{\circ}$ afterwards). We will therefore regard this $B_{\text {tor }}$ component as dominant, so that the star has a tendency for $\chi$ to increase towards its minimum-energy state of $\chi=90^{\circ}$, and so to precess spontaneously. Purely toroidal fields are, however, unstable (Tayler 1973) - and so we assume the presence of a poloidal component $B_{\mathrm{pol}}$ weak enough to be neglected in the first instance, but strong enough to stabilise the overall $B$. Using a solution for $\epsilon_{B}$ of a toroidal field (Lander \& Jones 2009), we may then calculate:

Po $=\frac{\Omega_{\mathrm{p}}}{\Omega_{0}}=\frac{\Omega_{0}\left|\epsilon_{B}\right| \cos \chi}{\Omega_{0}}=3 \times 10^{-6}\left(\frac{B_{\text {tor }}}{10^{15} \mathrm{G}}\right)^{2}$

clearly far smaller than in the fluid-dynamics context.

Understanding how long the precession phase lasts requires a more detailed look at the dynamics of a young magnetised NS. Although its bulk motion is precession, within the star this must be supported by a complicated field of hydromagnetic motions $\dot{\xi}$ (Mestel \& Takhar 1972), with the first self-consistent solution being found by Lander \& Jones (2017). Secular viscous damping of $\dot{\xi}$ reduces the precessional kinetic energy, and thus causes the evolution of $\chi$ towards $90^{\circ}$ for our assumed dominantly-toroidal $B$ (Jones 1975). Solutions of the coupled $\Omega-\chi$ differential equations indicate that the phase of increasing $\chi$ happens around $100 \mathrm{~s}$ after birth, when the temperature $T \sim 10^{10} \mathrm{~K}$ (Lander \& Jones 2020).

Precession alone can amplify both components of $B$, but since we anticipate that $B_{\text {tor }}$ will already be large, we are most interested in how much $B_{\text {pol }}$ (potentially considerably weaker) can catch up. $B_{\text {pol }}$ is also the field component that extends beyond the star, connecting to the surface dipole value $B_{\text {dip }}$ we estimate from NS spindown, and whose factor-1000 range of strengths we wish to explain. The convection-like structure of $\dot{\xi}$ (see fig. 8 from Lander \& Jones (2017)) is already promising for dynamo action: simulations of fully-convective M stars show that the interplay of (uniform) rotation with relatively slow convection can lead to a strong large-scale axisymmetric $B$ (Browning 2008).

To understand the effect of possible precession-driven dynamo action on $B_{\text {pol }}$, we imagine taking the stellar model of Lander \& Jones (2017) (precessing, with a toroidal background field) and adding a seed poloidal field $\boldsymbol{B}_{\text {seed }}$, which will be passively advected by the fluid motion (on large scales given by $\dot{\boldsymbol{\xi}}$, and on small scales probably turbulent, given the large Reynolds number $\left.v_{\text {char }} l_{\text {char }} / v_{\mathrm{S}} \sim 10^{8}\right)$. $B_{\text {pol }}$ thus undergoes a kind of forced precession analogous to the set-up in previous work on precessiondriven dynamos. This is a reasonable first approximation as long as $B_{\mathrm{pol}}$ is small enough for the overall magnetic distortion to remain prolate, and for the effect on $\dot{\xi}$ to be negligible.

As a first step towards understanding this dynamo scenario, we will take the standard approach of considering its initial kinematic phase, where one can assume that a turbulent $\boldsymbol{v}$ drives magnetic-field amplification, but without considering the Lorentz force associated with this newly-created $B$. The small-scale turbulent $\boldsymbol{v}$ averages to the fluid precession solution discussed above, $\bar{v}=\dot{\xi}$, and in the kinematic limit the induction equation becomes:

$\frac{\partial \overline{\boldsymbol{B}}}{\partial t}=\nabla \times[\dot{\boldsymbol{\xi}} \times \overline{\boldsymbol{B}}-\eta \nabla \times \overline{\boldsymbol{B}}]$.

We plug into this equation an ansatz of an exponentially-growing mode, $\boldsymbol{B}_{\text {pol }}(\boldsymbol{r}, t)=\boldsymbol{B}_{\text {seed }}(\boldsymbol{r}) \mathrm{e}^{t / \tau_{\text {amp }}}$, where $\tau_{\text {amp }}$ the field amplification timescale. This yields:

$$
\frac{1}{\tau_{\mathrm{amp}}} \boldsymbol{B}_{\text {seed }}=\nabla \times\left(\dot{\boldsymbol{\xi}} \times \boldsymbol{B}_{\text {seed }}\right)-\nabla \times\left(\eta \nabla \times \boldsymbol{B}_{\text {seed }}\right)
$$

- an eigenvalue problem for $1 / \tau_{\mathrm{amp}}$, which we assume admits solutions with positive real part, corresponding to exponential (dynamo) growth of $B_{\mathrm{pol}}$. In such analysis one generally finds that dynamo action is only possible above a certain Rm, but $\eta$ is so small for NS matter that this will not be a limiting factor, and in this kinematic phase may be neglected. Now rearranging eq. (6) and using scalings from Lander \& Jones (2017), we find that:

$\tau_{\text {amp }}=\frac{B_{\text {seed }}^{2}}{\boldsymbol{B}_{\text {seed }} \cdot\left[\nabla \times\left(\dot{\boldsymbol{\xi}} \times \boldsymbol{B}_{\text {seed }}\right)\right]} \sim \frac{l_{\text {char }}}{v R_{*} \epsilon_{\Omega} \epsilon_{B} \cos \chi}$,

where $v=\Omega / 2 \pi$ is the rotation rate in $\mathrm{Hz}$ and $\epsilon_{\Omega}$ the centrifugal distortion. For a given seed field we can quantitatively calculate $\tau_{\text {amp }}$, since we also know $\dot{\boldsymbol{\xi}}$ (Lander \& Jones 2017). $\boldsymbol{B}_{\text {seed }}$ is probably highly model-dependent, however, so to maintain generality we will instead use the above approximation in terms of $l_{\text {char }}$.

We need $\tau_{\mathrm{amp}}$ to be short compared with the duration $\tau_{\chi}$ of the precession phase, which in our scenario is set by damping of $\dot{\xi}$ due to bulk viscosity. This effect is sensitive to $\Omega, B$ and $T$, but once $T \lesssim 10^{10} \mathrm{~K}$ the limiting case given by eq. 61 of Lander \& Jones (2018) becomes increasingly accurate. Using this result and eq. (7), we arrive at the following criterion for significant dynamo action:

$$
\begin{aligned}
1 \lesssim \frac{\tau_{\chi}}{\tau_{\mathrm{amp}}} \approx 20 & \left(\frac{1 \mathrm{~cm}}{l_{\mathrm{char}}}\right)\left(\frac{v}{100 \mathrm{~Hz}}\right)\left(\frac{10^{10} \mathrm{~K}}{T}\right)^{6}\left(\frac{B_{\mathrm{tor}}}{10^{15} \mathrm{G}}\right)^{4} \\
& \times \sin ^{2} \chi \cos \chi .
\end{aligned}
$$

We first note that if other quantities are close to the fiducial values we use, $l_{\text {char }} \lesssim 20 \mathrm{~cm}$ is required for an effective dynamo. Although the ratio increases rapidly for cooler stellar models, dynamo action will be stifled for $T \lesssim 10^{9} \mathrm{~K}$ when the core becomes superconducting; see next section. It is noteworthy that the dynamo depends only linearly on rotation, but strongly on $B_{\text {tor }}-$ which suggests that 


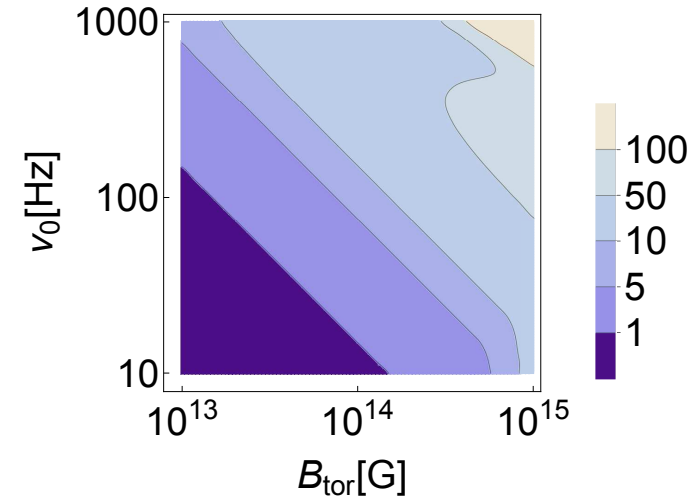

Figure 1. The maximum lengthscale (colourscale; in $\mathrm{cm}$ ) on which the precession-driven dynamo is effective, for a parameter space of models with different $B_{\text {tor }}, v_{0}$ as shown.

slight variations in (e.g.) the birth differential rotation could manifest themselves as the kind of factor-1000 differences we infer in $B_{\text {dip }}$.

As a more quantitative complement to eq. (8), let us demand $\tau_{\chi}=\tau_{\mathrm{amp}}$ and rearrange eq. (7) to give an expression for the threshold $l_{\text {char }}=l_{\text {char }}^{*}$ for an effective dynamo, as a function of $\tau_{\chi}, \Omega, \chi$. From the results of self-consistent time-evolutions of the coupled $\Omega-\chi$ equations (Lander $\&$ Jones 2020) we find $\tau_{\chi}$ and $\Omega, \chi$ timeaveraged over the $\chi$-evolution phase, for models with different $B_{\text {tor }}$ and birth rotational frequency $v_{0}{ }^{3}$. From these quantities we calculate $l_{\text {char, }}^{*}$, plotting the results in fig. 1 . For a wide range of models we find $l_{\text {char }}^{*}=1-50 \mathrm{~cm}$, in broad agreement with eq. (8). Generally speaking $l_{\text {char }}^{*}$ is proportional to $v_{0}$ and $B_{\text {tor }}$, as seen from the diagonal contours, but for $B_{\text {tor }}>10^{14} \mathrm{G}$ the relationship is more complex: $l_{\text {char }}^{*}$ is larger than expected for low $v_{0}$, and smaller than expected for high $v_{0}$. This is a manifestation of the nontrivial behaviour of viscous damping during the precession phase (Dall'Osso \& Perna 2017; Lander \& Jones 2018).

Dynamo action could end either through the usual mechanism of saturation - a backreaction of the newly-created $B$ on the flow generating it - or by precession ceasing, as reflected in the trigonometric dependences in $\tau_{\chi} / \tau_{\mathrm{amp}}$. The latter scenario will occur for $\chi \rightarrow 90^{\circ}$ (if $B_{\text {tor }}$ remains dominant) or $\chi \rightarrow 0^{\circ}$ (if the newlycreated $B_{\text {pol }}$ grows to become dominant, or if the star's external alignment torque is enhanced, e.g. by fallback matter). The most optimistic scenario would be for $B_{\mathrm{pol}}$ to grow large enough to arrest the evolution of $\chi$ at some intermediate angle, prolonging the precession phase and therefore the dynamo. Since this process ultimately taps rotational energy $E_{\text {rot }}$, a firm upper limit $B_{\max }$ for the increase in average $B$-field strength is given by equating its associated energy with $E_{\mathrm{rot}}$ and rearranging:

$B_{\max }=\sqrt{\frac{8 \pi E_{\mathrm{rot}}}{4 \pi R_{*}^{3} / 3}} \approx 4 \times 10^{15}\left(\frac{v}{100 \mathrm{~Hz}}\right) \mathrm{G}$.

Let us assume, as before, that the dynamo acts to amplify $B_{\text {dip }}$, since $B_{\text {tor }}$ is already large. Then the above estimate suggests that it is plausible to amplify a very weak $B_{\text {dip }}$ to magnetar strength, but reaching the value $B_{\max }$ would need ideal conditions. If the dynamo is ineffective, the resulting star would still have a strong $B_{\text {tor }}$ but a more typical pulsar-like $B_{\text {dip }} \sim 10^{12} \mathrm{G}$. We argued in the last section that a birth dynamo may be inhibited at high $\operatorname{Pr}_{m}$. Why

3 Using $B_{\text {dip }}=0.01 B_{\text {tor }}$, alignment torque prefactor $k=2$, and defining $\tau_{\chi}$ as the era for which $2^{\circ}<\chi<88^{\circ}$. should this precession-driven dynamo fare any better? Firstly, since $v_{\text {char }}=\dot{\xi} \sim 10-10^{3} \mathrm{~cm} \mathrm{~s}^{-1}$ is not so high (Lander \& Jones 2018), $\mathrm{Rm}$ is a comparatively modest $\approx 10^{12}$ for the precession phase. Perhaps more importantly though,

$\operatorname{Pr}_{\mathrm{m}}=2 \times 10^{5}\left(T / 10^{10} \mathrm{~K}\right)^{-4} \approx 10^{4}-10^{6}$

in this case (combining results from Baym et al. (1969) and Cutler \& Lindblom (1987)). Note that we calculate $\operatorname{Pr}_{\mathrm{m}}$ using shear viscosity and not the far stronger bulk viscosity; typically it is shearing rather than compressional motions that drive dynamo action. The above $\operatorname{Pr}_{\mathrm{m}}$ will still substantially slow down reconnection, but by a factor $10^{3}$ (using eq. (2)) rather than the proto-NS's $10^{8}$. Furthermore, since the precession phase typically lasts a factor $100+$ longer than the convective phase ${ }^{4}$, there is also less urgency for reconnection and an inverse cascade to amplify $B$.

\section{PHASE 3: THE COLD SUPERCONDUCTING STAR}

Very little work has considered the possibility of magnetic-field generation in the core of a mature NS, mainly because it seems unlikely the star undergoes the kind of fluid motion needed for a dynamo - it is, for example, not convectively unstable. Differential rotation might, however, persist into this late phase (Melatos 2012), and if $\chi$ is not very close to $0^{\circ}$ or $90^{\circ}$ precession is also possible.

If suitable fluid motions exist, the main obstacle to late-stage dynamo action is superconductivity of the core's protons. The critical temperature for NS superconductivity is density-dependent and poorly constrained, but generally in the range $(1-6) \times 10^{9} \mathrm{~K}$. At an age of roughly a month to a year, most of a NS's core will have cooled sufficiently to be superconducting (Ho et al. 2015).

Intrinsic to dynamo action is that on small enough scales magnetic-field lines must reconnect. In contrast with the case of normally-conducting matter, the field lines in the type-II superconducting NS core are associated with distinct physical structures: fluxtubes. In their equilibrium state these form an Abrikosov lattice with spacing of $3.5 \times 10^{-10}\left(B / 10^{12} \mathrm{G}\right)^{-1 / 2} \mathrm{~cm}$ (Mendell 1998). Although our understanding of the physics of $B$ in the superconducting core is still rudimentary, the dissipation (and therefore reconnection) timescale is expected to be substantially longer than in the normally-conducting state (Baym et al. 1969). The energy penalty for breaking a fluxtube and the distinct inter-fluxtube spacing both hinder reconnection, which we believe will only happen at the crust-core boundary, where superconductivity ceases. As a result, dynamo action within the core seems unlikely. A dim possibility remains, however, that fluid motions could act to bunch up fluxtubes enough for superconductivity to be destroyed locally, thus allowing for a dynamo in some region of limited size.

\section{OUTLOOK}

We have argued that powerful amplification of a NS's large-scale $B$ is difficult during both its birth and mature phases. Although the conditions in a proto-NS superficially resemble those of a classic dynamo, the high values of $\mathrm{Rm}$ and $\mathrm{Pr}_{\mathrm{m}}$ may lead to qualitatively different - and ineffectual - action. If so, proto-NSs would never attain magnetar-strength $B_{\mathrm{dip}}$, casting doubt on the viability of various models for e.g. $\gamma$-ray bursts and their afterglow light curves.

\footnotetext{
4 Using the code from Lander \& Jones (2020) for typical magnetar parameters; for extremely high $\Omega$ the precession phase is shortened.
} 
Magnetic-field amplification is required at some stage, however, and this paper introduces a potentially promising new mechanism for doing so: a precession-driven dynamo acting $\sim 100 \mathrm{~s}$ after birth. This could bypass some of the problems associated with the proto-NS phase, may be a universal feature of a NS's early evolution, and can naturally explain the observed large variation of $B_{\text {dip }}$ in young NSs. If $\chi$ evolution stalls once the dynamo stops, there is an intriguing possibility of inferring a NS's internal magneticfield geometry (whether it is dominantly poloidal or toroidal) from measurements of its present-day $\chi$.

The ideas outlined here are, however, clearly preliminary. They could become considerably more plausible through work on two key issues: the hydromagnetic dynamics of the precession phase, and the development of $B$ during and beyond the kinematic phase of the dynamo. Both of these will require numerical simulations.

There are a number of hints from observations that a magnetar's interior field may be considerably stronger than its external one: Makishima et al. (2021) argue that long-term modulation in the pulse profile of a few magnetars can be explained by precession, if $B_{\text {tor }} \approx 10^{16} \mathrm{G} \approx 100 B_{\text {dip }}$, and Granot et al. (2017) suggests that $B_{\text {tor }} \gtrsim 30 B_{\text {dip }}$ for the magnetar Swift J1834.9-0846. If $B_{\text {tor }}$ is so high, equation (8) suggests that amplification of $B_{\text {dip }}$ during a precession-driven dynamo should have been relatively efficient, but the observed $B_{\text {dip }} \approx 10^{14} \mathrm{G}$ are rather lower than expected values of $B_{\max }$ from equation (9). One possibility is that the dynamo saturates for a poloidal field of $\sim 10^{14} \mathrm{G}$.

The puzzling nature of the central compact objects, very young NSs with $B_{\text {dip }} \sim 10^{10} \mathrm{~K}$ (Halpern \& Gotthelf 2010), could be interpreted as the result of a failed precession-driven dynamo. Even if $B_{\text {tor }}=10^{15} \mathrm{G}$, say, $\chi$ could be kept small by a strong torque due to fallback matter, thus limiting any precession-driven amplification of $B_{\text {dip }}$; if so, the $\chi$ of these objects should still be small today.

Previous work on precession-driven dynamos has focussed on a fluid coupled to a precessing container; in the context of the Earth, its crust. By contrast, we have argued that a similar effect could act in a magnetised fluid star, and therefore many of the ideas presented here could also be viable for explaining long-term field (re)generation in main-sequence oblique rotators.

\section{ACKNOWLEDGEMENTS}

I thank Jonathan Granot for interesting correspondence on some of these ideas, and the referee for a very detailed and insightful report that substantially improved this paper.

\section{DATA AVAILABILITY}

The specific data underlying this article, and additional data for other related models, will be made available upon reasonable request.

\section{REFERENCES}

Baym G., Pethick C., Pines D., 1969, Nature, 224, 674

Bonanno A., Rezzolla L., Urpin V., 2003, A\&A, 410, L33

Brandenburg A., 2001, ApJ, 550, 824

Brandenburg A., Rempel M., 2019, ApJ, 879, 57

Browning M. K., 2008, ApJ, 676, 1262

Bucciantini N., Del Zanna L., 2013, MNRAS, 428, 71

Bullard E. C., 1949, Proceedings of the Royal Society of London Series A, 197,433
Cutler C., Lindblom L., 1987, ApJ, 314, 234

Dall'Osso S., Perna R., 2017, MNRAS, 472, 2142

Donati J. F., Landstreet J. D., 2009, ARA\&A, 47, 333

Epstein R. I., 1979, MNRAS, 188, 305

Finn J. M., Ott E., 1988, Physics of Fluids, 31, 2992

Frisch U., Pouquet A., Leorat J., Mazure A., 1975, Journal of Fluid Mechanics, 68, 769

Galloway D. J., Proctor M. R. E., Weiss N. O., 1978, Journal of Fluid Mechanics, 87, 243

Giesecke A., Vogt T., Gundrum T., Stefani F., 2018, Phys. Rev. Lett., 120, 024502

Graber V., Andersson N., Glampedakis K., Lander S. K., 2015, MNRAS, 453,671

Granot J., Gill R., Younes G., Gelfand J., Harding A., Kouveliotou C., Baring M. G., 2017, MNRAS, 464, 4895

Halpern J. P., Gotthelf E. V., 2010, ApJ, 709, 436

Ho W. C. G., Elshamouty K. G., Heinke C. O., Potekhin A. Y., 2015, Phys. Rev. C, 91, 015806

Jafari A., Vishniac E. T., Kowal G., Lazarian A., 2018, ApJ, 860, 52

Janka H. T., Moenchmeyer R., 1989, A\&A, 226, 69

Jones P. B., 1975, Astro. Space Sci., 33, 215

Jones D. I., Andersson N., 2001, MNRAS, 324, 81

Keil W., Janka H. T., Mueller E., 1996, ApJ, 473, L111

Krause F., Raedler K. H., 1980, Mean-field magnetohydrodynamics and dynamo theory

Lander S. K., Jones D. I., 2009, MNRAS, 395, 2162

Lander S. K., Jones D. I., 2017, MNRAS, 467, 4343

Lander S. K., Jones D. I., 2018, MNRAS, 481, 4169

Lander S. K., Jones D. I., 2020, MNRAS, 494, 4838

Landstreet J. D., 1992, A\&ARv, 4, 35

Lazarian A., Vishniac E. T., 1999, ApJ, 517, 700

Makarenko E. I., Igoshev A. P., Kholtygin A. F., 2021, MNRAS, 504, 5813

Makishima K., Enoto T., Yoneda H., Odaka H., 2021, MNRAS, 502, 2266

Malkus W. V. R., 1968, Science, 160, 259

Masada Y., Takiwaki T., Kotake K., 2020, arXiv e-prints, p. arXiv:2001.08452

Melatos A., 2012, ApJ, 761, 32

Mendell G., 1998, MNRAS, 296, 903

Mestel L., Takhar H. S., 1972, MNRAS, 156, 419

Moffatt H. K., 1978, Magnetic field generation in electrically conducting fluids

Moffatt H. K., Proctor M. R. E., 1985, Journal of Fluid Mechanics, 154, 493

Mösta P., Ott C. D., Radice D., Roberts L. F., Schnetter E., Haas R., 2015, Nature, 528, 376

Obergaulinger M., Cerdá-Durán P., Müller E., Aloy M. A., 2009, A\&A, 498, 241

Parker E. N., 1992, ApJ, 401, 137

Raynaud R., Guilet J., Janka H.-T., Gastine T., 2020, Science Advances, 6 , eaay 2732

Reboul-Salze A., Guilet J., Raynaud R., Bugli M., 2021, A\&A, 645, A109

Rincon F., 2019, Journal of Plasma Physics, 85, 205850401

Sawai H., Yamada S., Suzuki H., 2013, ApJ, 770, L19

Spitzer L. J., 1958, in Lehnert B., ed., Vol. 6, Electromagnetic Phenomena in Cosmical Physics. p. 169

Spruit H. C., 2002, A\&A, 381, 923

Stella L., Dall'Osso S., Israel G. L., Vecchio A., 2005, ApJLett., 634, L165

Sukhbold T., Ertl T., Woosley S. E., Brown J. M., Janka H. T., 2016, ApJ, 821,38

Tayler R. J., 1973, MNRAS, 161, 365

Thompson C., Duncan R. C., 1993, ApJ, 408, 194

Thompson T. A., Chang P., Quataert E., 2004, ApJ, 611, 380

Tilgner A., 2005, Physics of Fluids, 17, 034104

Tilgner A., 2007, Geophysical and Astrophysical Fluid Dynamics, 100, 1

Vainshtein S. I., Cattaneo F., 1992, ApJ, 393, 165

Vă̈nshteĭ S. I., Zel'dovich Y. B., 1972, Soviet Physics Uspekhi, 15, 159

Wu C.-C., Roberts P., 2009, Geophysical and Astrophysical Fluid Dynamics, 103,467

Zhang B., Mészáros P., 2001, ApJ, 552, L35 